\title{
Médecine sans parole
}

Les étudiants sont éduqués avant tout de façon virtuelle et plus tard, ils délèguent au personnel soignant la parole et l'écoute. Les examens à choix multiple et les systèmes de diagnostic par ordinateur font partie d'une technologie de haut niveau où la parole disparaît. Les calculateurs produisent des réalités virtuelles, dont les données «dures» représentent bien plus que celles, «douces», d'une conversation. Si les médecins laissent à leurs appareils le soin de tout percevoir, ils éliminent leur propre personne. Ça ne serait pas si grave si l'on ne risquait pas de voir les malades eux-mêmes disparaître dans leur monde et se taire. La médecine bien comprise demande une langue naturelle et élaborée à la fois. Où peut-on l'apprendre? Nous avons tous ou avons tous eu un jour dans les études un déficit de parole, étions des amateurs et autodidactes, dans le meilleur des cas dotés d'un talent naturel, évoluant entre la médecine de pointe et la communication de l'âge de la pierre.

Ces vingt dernières années, un «tournant narratif» s'est imposé dans de nombreux domaines de la science comme nouvelle méthode de connaissance. La narration et l'analyse de textes narratifs sont une manière fructueuse de saisir comment les humains se conçoivent eux-mêmes, comment l'individu travaille à sa biographie et à son identité et quels sont les rôles joués par la culture et la société dans ce processus. Ce qui est vécu est transposé en motifs et en modèles narratifs qui déterminent à leur tour ce qu'est l'expérience. Le patient et le médecin «construisent» leur relation selon des objectifs personnels et une logique de production. Les deux racontent une histoire en suivant des principes de construction spécifiques. Le patient structure son expérience subjective et le médecin l'interprète selon les modèle d'interprétation qu'il a appris. L' «histoire du malade» qui en ressort allie, dans le cas idéal, un savoir d'expert à l'interprétation subjectivo-existentielle des troubles exposés. Même si le médecin ignore le vécu de la maladie et les bases de l'action de celle-ci, il est soumis à son pouvoir et à son effet. En revanche, ce qui peut être abordé de manière cognitive trouve une résonance au niveau des émotions et permet de définir ensemble des possibilités d'agir et des perspectives d'avenir. Le processus de guérison est alors facilité et devient peut-être même seulement possible à ce moment-là. La nécessaire compétence à parler développe la sensibilité professionnelle et la faculté d'empathie requises.

De nombreux articles de revues médicales soulignent les avantages de la «médecine narrative». Dans «Primary Care» [1], on a pu lire à de nombreuses reprises la fonction essentielle du récit, de même que dans une longue recension d'article dans la «Revue Médicale Suisse», intitulée «Quelle place pour l'écriture dans le milieu médical?» [2] et soulignant l'importance de la subjectivité du thérapeute et du patient en tant que complément négligé de la médecine des appareils. Le plus simple est souvent le plus compliqué, si l'on en croit l'anecdote suivante concernant un oncologue. Lorsqu'il demanda à une patiente quel conseil le plus important il devrait donner lors d'une allocution de fin d'études médicales, elle répondit: «Dites-leur de frapper à ma porte, de me saluer et de me dire au revoir, en me regardant dans les yeux quand ils me parlent!»

Ne nous lassons pas de le dire: si le médecin ne se préoccupe pas de son langage, il ne fait pas qu'y perdre, il perd aussi une grande source de plaisir. Un petit pas suffit, que l'écrivain allemand Ralf Rothmann décrit très exactement: «Un indescriptible bonheur, même s'il commence souvent dans la grisaille comme une torture qui fait du bien: écrire. Ne pas avoir la bougeotte et laisser vagabonder son esprit, telle est l'attitude à adopter. [...] Soudain, le mot tombe et fait du texte un sujet jamais vu, formidable, hésitant, tiraillé jusqu'à la déchirure entre ce qu'on veut exprimer et ce que la langue veut, un être vivant... et le monde est tout à coup à la bonne place. On jette son crayon et on embrasse de joie le chambranle de la porte.» [3]

\section{Erhard Taverna}

\section{Littérature}

1 www.primarycare.ch.

2 Louis-Courvoisier M. Quelle place pour l'écriture dans le milieu médical Rev Med Suisse. 2005.

3 Rothmann R. Stier. Roman. Frankfurt: Suhrkamp Taschenbuch 2255; 1993. 